\title{
Effects of National Gymnastics and Brain Gymnastics on Frontal Lobe Activity
}

\begin{abstract}
The effects of national gymnastics and brain gymnastics on brain activity were analyzed in 20 healthy university students. The students were assigned to either a national gymnastics group or a brain gymnastics group. Their frontal lobe activity was recorded before and after the exercise, and the resting, attention, and concentration indexes of brain waves were measured.

The resting index significantly decreased and concentration index significantly increased(p<.05) after the exercise in both the national and brain gymnastics groups. However, the attention index significantly increased only after brain gymnastics, and, on the contrary, decreased after national gymnastics.
\end{abstract}

These findings suggest that brain gymnastics is effective for improving attention.

Key words: National Gymnastics; Brain Gymnastics; Attention Index; Concentration Index

\author{
Kyung Hee Jeon \\ Chunnam Techno University, Gwangju, Korea \\ Received : 8 July 2015 \\ Revised : 12 August 2015 \\ Accepted : 17 September 2015 \\ Address for correspondence \\ Kyung Hee Jeon, PT. Ph.D \\ Department of Physical Therapy, \\ Chunnam Techno University, 51, \\ Cheomdan venture so-ro, Buk-gu, \\ Gwangju, Korea \\ Tel: 82-61-360-5132 \\ E-mail: mania1112@hanmail.net
}

\section{INTRODUCTION}

Mental activities such as learning, thinking, memory, and creation are not limited to the brain function but involve both the brain and body. For example, to stimulate brain function, the body needs to move(1). Body movement, which is a combination of internal and external activity of the nervous system, not only develops the neural network, internalizes learning in the neural network, and stimulates brain action but also contributes to cognitive function and emotional development(2). Human movements mostly result from brain activity, and those occurring after exercise affect brain activity via diverse pathways. The frontal lobe plays an important role in higher mental function and movement. It comprises the prefrontal lobe, which is related to performance of high mental functions, and primary motor area, premotor area, supplemental motor area, frontal eye area, and motor speech area, which are involved in conscious movement(3).

The frontal lobe is the center for processing activities like willingness and planning to move, generation and selection of programs, their exe- cution order and implementation, output signals, and motor feedback modification order(4). The frontal lobe accounts for about $40 \%$ of human cerebral cortex(5), and the functions of the frontal lobe may be largely classified into execution function and motor function. Execution function refers to the ability to form abstract concepts, maintain cognition, organize information in occupational memory, selectively inhibit inappropriate responses, and behave in a socially acceptable manner(6). Motor function refers to the ability to inhibit reflective behaviors, plan and control complex movements, and refine diverse movements. To regulate such behaviors, the frontal lobe exchanges information with almost all cortical and subcortical areas $(7,8)$. Such execution functions of the frontal lobe are related to attention and concentration(9).

Attention is an action and a cognitive process of selective concentration that subjectively or objectively ignores other sensory information while selecting necessary information(10). Concentration is a condition of focusing on and being engrossed in just one thing and, in many cases, refers to a concept pertaining to the orientation of energy. 
Concentration refers to an individual's mental power to pay focused attention to a chosen stimulus and is a term particularly used along with attention(especially, selective attention)(11). Attentive concentration affects cognitive process, task performance ability, and learning ability. An example of deteriorating attentive concentration is the increasing number of students whose attentive concentration is being affected by the popularization of smart phones, the Internet, and image data(12).

Children with attention deficit hyperactivity disorder(ADHD) exhibit lack of attention and impulsive and excessive behaviors because of lack of self-control. ADHD is caused by a functional disorder of the frontal lobe, among other neurophysiological causes, according to studies conducted using functional magnetic resonance imaging, single photon emission computed tomography, positron emission tomography, and electroencephalography(13, 14). Human brain activity generates brain waves. In brief, exchange of information occurs among cortical pyramidal cells at the synapse, generating fine electrical impulses. Brain waves are nothing but synchronized electrical impulses produced during brain activity. Analysis of frequencies generated by brain activity may provide information on brain activity. As main frequency ranges, the brain generates alpha waves during rest, the sensorimotor rhythm(SMR) waves when paying attention to surrounding conditions, and low beta or high beta waves when performing tasks or concentrating; the analyses of these waves provides the resting index, attention index, and concentration index, respectively(15).

Body movements affect brain functions by giving diverse stimuli to the entire brain. Many studies have reported that body movements support nerve cells, aid their generation, and activate nutrition supply, thereby improving cognitive functions(16); stimulate the hippocampus (an area responsible for memory); generate new nerve cells in the dentate gyrus (17); generate neurotransmitters like brain derived neurotrophic factors(18) and, thereby, prevent depression(19); increase brain blood flow(20); and prevent brain tissue loss and reduce the incidence of Alzheimer's disease and agerelated dementia(21).

Brain gymnastics, introduced and developed by Dennison, is a simple physical exercise devised to strengthen brain functions. Created with an aim to reduce stress generated when learning and for developing learning potential to the maximum extent possible, brain gymnastics is used to improve learning effects by activating the brain to integrate the body and both the hemispheres of the brain $(2,22)$. Brain gymnastics increases attention of a patient who has experienced stroke(3) and controls learning stress by stimulating cerebral cortex activity(1). A brain development program, similar to brain gymnastics conducted for the entire body, was reported to influence various aspects of intelligence(23). With this background, the present study was conducted to examine the effects of national gymnasticswhich is often implemented in elementary and secondary schools and those of brain gymnasticswhich has diverse effects on attention and concentration of the frontal lobe through the measurement and analysis of brain waves and to assess the usefulness of these 2 techniques.

\section{METHODS}

\section{Subjects}

The study participants included 20 healthy girls and boys in their 20s, free from any neurokinematic or cognitive function abnormalities. They were assigned to either a national gymnastics group or a brain gymnastics group. The 2 groups were confirmed to match for sex and age distribution (p >.05)(Table 1).

Table 1. Subject demographics

\begin{tabular}{ccc}
\hline & National Gymnastics & Brain Gym \\
\hline $\mathrm{N}$ & 10 & 10 \\
Age $(\mathrm{yr})$ & $22.21 \pm 1.13$ & $22.40 \pm 1.50$ \\
Height $(\mathrm{cm})$ & $166.96 \pm 7.51$ & $167.30 \pm 7.64$ \\
Weight $(\mathrm{kg})$ & $60.50 \pm 8.66$ & $61.10 \pm 9.57$ \\
\hline
\end{tabular}

\section{Study Design and Method}

The brain waves of the 20 participants were recorded before and after the exercise. The resting, attention, and concentration indexes before and after the exercise were measured and compared within and between the groups.

\section{Measurement Tool}

The resting, attention, and concentration indexes were measured and analyzed using Neuroharmony 
(Braintech, Korea), a non-invasive, a 2 channel brain wave measuring and training device, structured like a head band. It measures brain waves of the left and right side of the brain simultaneously at the Fp1 and Fp2 of the prefrontal lobe according to the standard international 10-20 system by using sequential bipolar montage. Its reliability has been verified: the coefficient of brain wave values was $\mathrm{p}<.001$, as derived by comparing coefficients between each brain wave obtained using the Grass Neurodata Amplifier System(USA), a brain wave measuring device currently in rampant use.

\section{Exercise Method}

National gymnastics was introduced to national organizations and schools as "12 Kinds of New People's Gymnastics Method" in 1977 by the Korean government. Many schools still conduct national gymnastics, and some middle and high schools use it for performance appraisal programs. The 12 kinds of motions are, in the given order: warm up exercise, leg exercise, arm exercise, neck exercise, chest exercise, side exercise, back and abdominal exercise, trunk exercise, whole body exercise, leg exercise, arm and leg exercise, and taking a breath exercise.

Brain exercise was applied by selecting eight motions introduced as activities that closely connect the brain function and body function, based on brain gymnastics devised and developed by Dennison. The brain exercise included the following: brain button, owl, hook ups, double doodle, lazy 8s, cross crawl, cross crawl sit ups, and the cross crawl step.

\section{Data Analysis}

After national gymnastics and brain gymnastics, brain waves were measured from Fp1 and Fp2 using Neuroharmony, and resting, attention, and concentration indexes were measured. SPSS 18.0 version was used for the analysis of brain wave data. A paired samples t-test was used to analyze changes before and after national gymnastics and brain gymnastics, and an independent samples ttest was used to determine differences between the 2 groups. Significance level was set at $\alpha=$ .05 .

\section{RESULTS}

\section{Within-Group Comparison of the Measured Indexes Before and After the Exercise}

Differences in the resting, attention, and concentration indexes before and after the exercise were analyzed to evaluate the gymnasticsinduced changes in brain activity. In the national gymnastics group, the resting index, attention index, and concentration index before and after the exercise were $30.8 \pm 5.45$ and $25.3 \pm 4.00$, $21.1 \pm 3.35$ and $17.4 \pm 3.06$, and $4.1 \pm 4.48$ and $27.0 \pm 6.16$, respectively. The resting and attention indexes significantly decreased whereas the concentration index significantly increased after the exercise when compared with values before the gymnastics $(p<.05)$. In the brain gymnastics group, the resting index, attention index, and concentration index before and after the gymnastics were $29.3 \pm 4.72$ and $25.1 \pm 6.72,21.2 \pm 4.49$ and

Table 2. Differences in brain wave values before and after the exercise in the national gymnastics group and the brain gymnastics group

\begin{tabular}{cccccccc}
\hline \multirow{2}{*}{ Brain Waves } & \multicolumn{3}{c}{ National Gymnastics } & \multicolumn{3}{c}{ Brain Gymnastics } \\
\cline { 3 - 7 } & & $\mathrm{M} \pm \mathrm{SD}$ & $\mathrm{t}$ & $\mathrm{p}$ & $\mathrm{M} \pm \mathrm{SD}$ & $\mathrm{t}$ & $\mathrm{p}$ \\
\hline \multirow{2}{*}{ Resting } & Before exercise & $30.8 \pm 5.45$ & 2.509 & $.033^{*}$ & $29.3 \pm 4.72$ & 2.449 & $.037^{*}$ \\
& After exercise & $25.3 \pm 4.00$ & & & $25.1 \pm 6.72$ & & \\
\multirow{2}{*}{ Attention } & Before exercise & $21.1 \pm 3.35$ & & & & & \\
& After exercise & $17.4 \pm 3.06$ & 4.772 & $.001^{* *}$ & $21.2 \pm 4.49$ & -3.951 & $.003^{* *}$ \\
& Before exercise & $24.1 \pm 4.48$ & & & $27.4 \pm 3.75$ & & \\
& After exercise & $27.0 \pm 6.16$ & -3.097 & $.013^{*}$ & $22.6 \pm 4.93$ & -3.633 & $.005^{* *}$ \\
\hline
\end{tabular}

${ }^{*} \mathrm{P}\left\langle 05,{ }^{* *} \mathrm{P}<01\right.$ 
$27.4 \pm 3.75$, and $22.6 \pm 4.93$ and $28.5 \pm 6.54$, respectively. The resting index significantly decreased whereas the average values of attention and concentration indexes significantly increased after the gymnastics when compared with the values before the gymnastics $(\mathrm{P}<.05$; Table 2$)$.

\section{Between-Group Comparison of Changes in Indexes, and Brain Conditions, Before and After the Exercise}

To compare changes in the brain conditions between the 2 groups after the national gymnastics and brain gymnastics, between group differences in changes in the resting, attention, and concentration indexes were analyzed. In the national gymnastics and brain gymnastics groups, the resting indexes were $-5.50 \pm 6.93$ and -3.6 \pm 4.65 , respectively, the attention indexes were $-3.70 \pm 2.45$ and $6.2 \pm 4.96$, respectively, and the concentration indexes were $2.90 \pm 2.96$ and $6.7 \pm 5.83$, respectively. The resting values decreased in both national gymnastics and brain gymnastics groups after the exercise. The attention values however decreased in the national gymnastics group but increased in the brain gymnastics group after the exercise. The concentration index increased in both the groups. The between group $\mathrm{t}$ value and $\mathrm{P}$ value for resting were -.720 and .481, respectively, showing no significant difference. The between group $t$ value and $\mathrm{P}$ value for attention were significant at 5.656 and .000 , respectively ( $\mathrm{p}<.05$ ), with the attention index of the brain gymnastics group being higher. The between-group $t$ value and $\mathrm{P}$ value for concentration were -1.837 and .083 , respectively, showing no significant difference. The concentration index increased in both the groups, but the increase was higher in the brain gymnastics group (Table 3).

Table 3. Between-group comparison of changes in brain wave indexes before and after the exercise

\begin{tabular}{|c|c|c|c|c|}
\hline \multicolumn{2}{|c|}{ Group } & \multirow{2}{*}{$\begin{array}{r}\text { Mean } \pm S D \\
-5.5 \pm 6.93\end{array}$} & \multirow{3}{*}{$\frac{t}{-720}$} & \multirow{3}{*}{$\frac{p}{.481}$} \\
\hline \multirow{2}{*}{ Resting } & National Gymnastics & & & \\
\hline & Brain Gymnastics & $-3.6 \pm 4.65$ & & \\
\hline \multirow{2}{*}{ Attention } & National Gymnastics & $-3.7 \pm 2.45$ & \multirow{2}{*}{-5.656} & \multirow{2}{*}{$.000^{*}$} \\
\hline & Brain Gymnastics & $6.2 \pm 4.96$ & & \\
\hline \multirow{2}{*}{ Concentration } & National Gymnastics & $2.9 \pm 2.96$ & \multirow{2}{*}{-1.837} & \multirow{2}{*}{$.083^{*}$} \\
\hline & Brain Gymnastics & $6.7 \pm 5.83$ & & \\
\hline
\end{tabular}

${ }^{*} \mathrm{P}<.05,{ }^{* *} \mathrm{P}<.01$

\section{DISCUSSION}

We measured brain waves from the frontal lobe of 20 university students, analyzed differences in brain wave activity patterns, and examined the effects of national gymnastics and brain gymnastics on the frontal lobe activity of the brain. Our brain generates substantial alpha waves during rest, representing a state of being awake but not active. Further, the higher the resting index, the more deactivated the brain is. The resting value measured in this study decreased after the exercise when compared with before the exercise in both national gymnastics and brain gymnastics groups, suggesting that both the exercises stimulated brain activity.

SMR waves appear when the brain is awake at rest and is in attention, whereas $\beta$ waves appear when the brain is in the active condition. Here, the SMR wave is a mostly generated under the condition of complete attention/concentration, I.e., when the brain is in attention and concentration but not in tension. It refers to the brain's ability to deal with work by using lesser energy than in the case with $\beta$ waves. In this study, the attention index is related to the activity of SMR wave. The attention index of the national gymnastics group decreased whereas that of the brain gymnastics group increased after the exercise when compared with the values before the exercise. This result shows that national gymnastics decreases attention( $p<.05)$ but brain gymnastics cause an increase in attention $(p<.05)$. Concentration is represented by the appearance of low beta waves. The beta wave appears when the brain concentrates on an activity or is in a working 
state. Low beta waves are prevalent during thinking activity accompanied with mental load that requires concentrating on one topic such as when performing a calculation, mental or otherwise. The attention index significantly increased in both the groups after the exercise ( $p<.05)$, but the brain gymnastics showed better concentration. This is consistent with the results of a study by Lim who asserted that brain gymnastics had a positive effect in improving attention and concentration and self adjustment ability, and those of a study by Kim and Min who reported that brain stimulation therapy conducted for 6 weeks affected brain wave activity pattern, in particular, attention(23, 24, 25).

In a study by Shin and Son on the effects of exercise on the brain, they noted that single photon emission computed tomography applied to elderly women who performed dance sports showed that blood flow increased in the frontal lobe and the temporal lobe(26). When activation signals by a simple motor task and a complex motor task are compared, activation signals are higher and the areas are wider in the premotor area and the supplementary motor area during a complex motor task, suggesting a greater association of the premotor area and the supplementary motor area with a complex task. Participation in regular exercise led to an increase in synaptic plasticity after exercise and improvement in brain function by increasing connection of nerve cells to the dendritic processes and improvement in efficiency of the central nervous system function, thereby enhancing brain function. Complex exercise, resistance exercise, and aerobic exercise trigger positive plastic changes in the central nervous system, and changes in brain activation, according to the characteristics of exercise in the order of complex exercise, resistance exercise, and aerobic exercise, were effective in improving brain function and preventing brain blood vessel diseases(27). In addition, exercise improved academic performance; increased students' attention and concentration, and calculation ability; and contributed to selective attention and concentration(28, 29, 30, 31, 32).

Thus, exercise not merely increases blood flow and brain activation but also enhances cognitive ability, attention and concentration, and learning ability. To sum up many previous studies, exercise affects attention and concentration, thereby enhancing learning efficiency, but brain gymnastics is more effective for attention and concentra- tion than national gymnastics and may effectively contribute to improvement in learning ability of children whose attention and concentration ability has been degraded.

\section{CONCLUSION}

The study aimed to examine the effects of national gymnastics and brain gymnastics on brain activity pattern. Twenty university students were assigned to either a national gymnastics group or a brain gymnastics group, and the brain wave indexes were compared before and after the exercise. This study reached the following conclusions.

1. both the groups showed a decrease in their resting index, indicating brain activation.

2. only brain gymnastics showed an improvement in their attention.

3 . both groups showed improvement in concentration, but the improvement was greater after brain gymnastics.

Thus, both national gymnastics and brain gymnastics contribute to enhancing concentration, but brain gymnastics is more effective in increasing attention.

\section{REFERENCES}

1. Jeong JJ. The Brain Gym as a Brain-Based Teaching and Learning Strategies for the Students with Developmental Disabilities. Kor J Child Edu 2006; 15(4): 57-72.

2. Dennison PE, Dennison GE. Braingym teacher's edition(Revised). Califonia, Edu-Kinesthetics 1989.

3. Lee WT, Park KA. Medical Neuroanatomy, Korea medical books 2008.

4. Kandel E, Schwartz J, Jessell T, Siegelbaum S. Hudspeth A. Principles of Neural Science, Medical(5th ed). McGraw-Hill Education 2012.

5. Hynd GW, Willis G. Neurological foundations of intelligence: Handbook of intelligence: Theories, measurements, and applications. New York, Wiley 1985.

6. Miller T, Cumming P. Deficits in strategy application following frontallobe damage in man. Behavioral Brain Research 1991; 114(2): 727-741. 
7. Yeom JY. Cognitive changes in the frontal lobes across the lifespan. Hallym University 2006.

8. Lee SK. The effects of combined exercise on physical fitness, neurotransmitter, EEG and frontal lobe executive function in children with attention-deficit hyperactiviry disorder(ADHD). Sookmyung Women's University 2011.

9. Goldman-Rakic PS. Specification of higher cortical functions. Journal of head trauma Rehabilitation 1993; 8: 13-23.

10. Anderson JR. Cognitive psychology and its implications(6th ed). Worth Publishers 2004, pp.519.

11. http://global.britannica.com/topic/attention

12. Hong MO. The Effects of Brain Gym on Distractive Behaviors of children with ADHD. Kongju National University 2011.

13. Bonelli RM, Cummings JL. Frontal-subcortical circuitry and behavior Dialogues Clin Neurosci 2007; 9(2): 141-151.

14. Giedd JN, Blumenthal J, Molloy E, Castellanos FX. Brain imaging of attention deficit/hyperactivity disorder. Ann N Y Acad Sci 2001; 931: 3349.

15. Park BU. Optimization of brain function due to correlate EEG bands. The Korean Society of Jungshin Science symposium 2004; 21: 103-108.

16. Colcombe S, Kramer AF. Fitness effects on the cognitive function of older adults: a meta-analytic study. Psychol Sci 2003; 14(2): 125-130.

17. Moon MH, Jeong JW, Huh YB, Kim JH, Ahn HK, Park C. Temporal change of calbindin-D28k immunoreactivity in the dentate gyrus of voluntary running mouse. Anatomy and Cell Biology 2006; 39(2): 119-127.

18. Pencea V, Bingaman KD, Wiegand SJ, Luskin MB. Infusion of brain-derived neurotrophic factor into the lateral ventricle of the adult rat leads to new neurons in the parenchyma of the striatum, septum, thalamus, and hypothalamus. Neurosci 2001; 21(17): 6706-6717.

19. Lawlor DA, Hopker SW. The effectiveness of exercise as an intervention in the management of depression: systematic review and meta-regression analysis of randomised controlled trials. BMJ 2001; 322(7289): 763-767.
20. Swain RA, Harris AB, Wiener EC, Dutka MV, Morris HD, Theien BE, Konda S, Engberg K, Lauterbur PC, Greenough WT. Prolonged exercise induces angiogenesis and increases cerebral blood volume in primary motor cortex of the rat. Neuroscience 2003; 117(4): 1037-1046.

21. Laurin D, Verreault R, Lindsay J, MacPherson K, Rockwood K. Physical activity and risk of cognitive impairment and dementia in elderly persons. Arch Neurol 2001; 58(3): 498-504.

22. https://en.wikipedia.org/wiki/Brain_Gym

23. Kim YT, Min KO. The effects of frontal lobe EEG activity pattern by Paul Dennison's Brain Gym. J Brain Edu 2010; 3(5): 1-26.

24. Kwak YJ. The Effects of Brain Development Program on Multiple Intelligence. J Brain Edu 2006; 2: 165-181.

25. Im MH. The Effects of Brain Gym Program on Attention and Self-Regulated Ability of Elementary. Gongju National University of Education 2008.

26. Shin CH, Son TY. Effect Of Dance Sports Treatment On Estrogen and Change Brain Blood Flow In Senior Women. Journal of KAHPERD 2004; 43(4): 405-416.

27. Han JG. The variation of hormone related to aging and brain activation as per training type of old women. Chungang University 2007.

28. Song JW. Effect of early morning physical activity on Academic Achievement, numeracy, concentraton, and learning attitude of elementary school boys. Chonbuk National University 2012.

29. Jung HY. Effects of 10-week early morning exercise on physical activity and cognitive function in the middle school students. Sangmyung University 2013.

30. Kim JY. The effect of morning physical activity on physical ability and attention of school girls. Korea University 2014.

31. Ratey JJ, Hagerman RD. The Revolutionary New Science of Exercise and The Brain. Brown and Company 2008.

32. Kang BG. Memory and Synaptic Plasticity. J brain Soc 2001; 1(1): 13-24. 